\title{
On the Detection of Step Edges in Algorithms Based on Gradient Vector Analysis
}

\author{
A. Larré, E. Montseny \\ Computer Engineering Dept. Universitat Rovira i Virgili \\ Carretera de Salou s/n 43006 Tarragona, Spain \\ Email: alarre@etse.urv.es
}

\begin{abstract}
The study presented in this paper is an analysis for finding what is the minimum signal-to-noise ratio that must have a contour in order to allow its detection. For finding this minimum, all the study has been based on the analysis of the gradient vector, because this kind of algorithms are those which obtain best results. It can be concluded that: a) The analysis of the gradient vector argument is more robust than the module; b) A contour must have a signal-to-noise ratio greater than $4 / 5$ in order to be detected, if small operators are used to obtain the gradient vector, and so a good contour location be assured.
\end{abstract}

\section{Introduction}

Since the beginning of 60 's, many contour extractor algorithms have been developed. A contour in an image allows to obtain a very important feature of the objects in the scene: the shape of these objects. This feature is used to get an object description for its later recognition [10].

At this moment, there is not a general rurpose edge detector algorithm useful for any kind of images independently of the scene characteristics. There are many factors who affect the contour obtaining process. Now, algorithms based on the 1st derivative are the most important.

In [6], Haralick states that one edge extractor is better than another if reducing the information of the image (that is, adding noise) preserves its behavior. Haralick states that any edge extractor obtains good results in absence of noise. The processing of noise is the main problem in modern edge extraction algorithms. So, Davis in [4] lists the main factors adding noise in the images. These factors are photon noise, defocusing and textural structure of the objects. For this reason the noise immunity of the contour extractor algorithms must be one of its most important features.

At present, there are some algorithms that permit a good contour extraction in noisy images. These algorithms are Canny [3], Deriche [5] and Marr-Hildreth [8], all of them based on the gradient vector and 2 nd directional derivative analysis.

This work studies when an edge can be detected in function of its signal-to-noise ratio (SNR) with different algorithms based on the gradient vector analysis. The section 2 of the paper gives the problem of edge detection depending on the noise and 
the contrast. In section 3 the influence of noise in the gradient vector is studied. Finally, in function of this noise, the minimum contrast to be detected is analyzed.

\section{Problem Definition}

V.S. Nalwa [9] defines an edge as a local discontinuity in the illumination function defining a scene. A discontinuity of the $n$th order is defined as a function whose $n$th

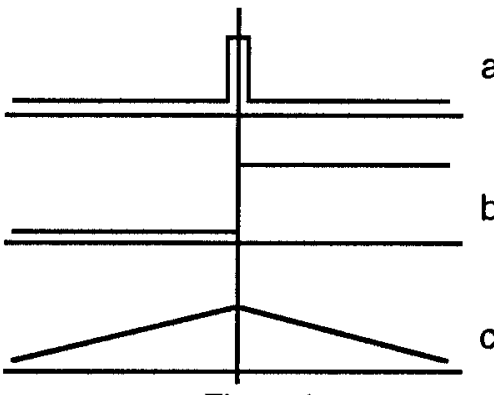

Figure 1 derivative contains a delta function. Davis [4] classified the edges of a scene in three classes according to their profile. These are: lines (discontinuity of order 0 ), figure 1a; steps (discontinuity of order 1 ), figure $1 \mathrm{~b}$; and roofs b (discontinuity of order 2), figure 1c. The discontinuities of greater orders are not relevant. The majority of algorithms attempt to solve the problem of extracting step edges as these are the ones that appear most frequently in any scene.

In real images, there are some factors that produce discontinuities in the illumination function, and they are not real contours. In the image of figure 2-a appear discontinuities associated to the noise, there are many discontinuities but they are very small. In figure 2-b appears discontinuities due to changes in the illumination of the scene. In figure 2-c, shows discontinuities due to real contours.

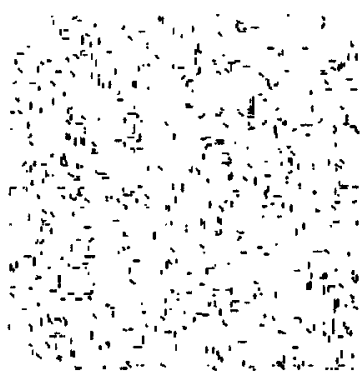

a

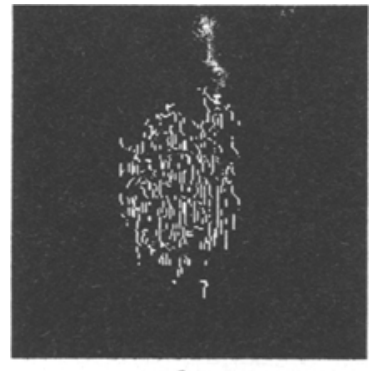

b

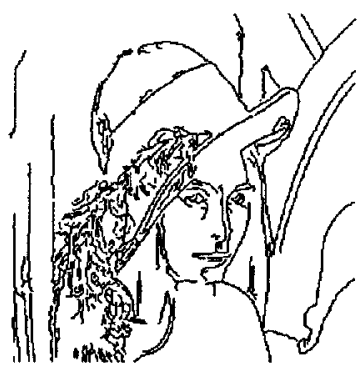

c

Figure 2

Haralick states that in images with a very low level of noise, all the edges can be detected with any algorithm. In this case contours with a very low contrast, can be detected. An example of this situation appears in figure 3-a. It has a circle at the center of the image with a very low contrast. Figure 3-b shows the contour extracted with Canny algorithm. But when the same image has a higher level of noise, like figure 3-c, contours with low contrast are more difficult to detect. Figure 3-d shows that the circle has not been detected. This example explains that the detection of a contour depends on its SNR. 


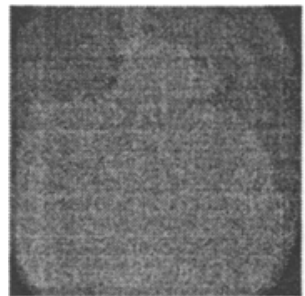

a

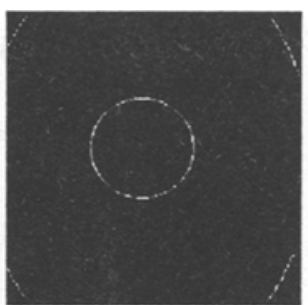

b

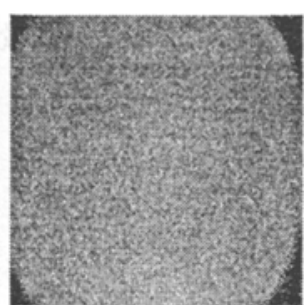

c

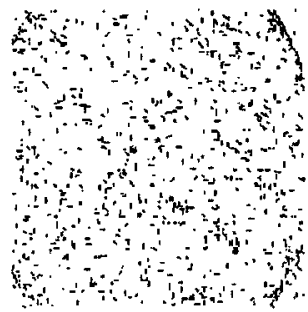

d

Figure 3

This work analyzes problems introduced by the noise, trying to find the minimum SNR that a real contour must have, in order to be detected.

\section{Analysis of the contour detection}

A contour in an image defines a discontinuity of the illumination function. Those arguments of the gradient vectors near of the contour change continuously along the contour and their modules have higher values than the rest of the image. For this reason, a contour can be detected with: a) the analysis of the gradient vector arguments, b) the analysis of the gradient vector modules, or c) the analysis of both, modules and arguments.

Because of the objective of this work is looking for the minimum SNR, it studies only straight step edges. For curved edges the SNR will be equal or higher.

In order to assure a good location of the contour, the operators for the gradient computation must be small. In the analysis made in this paper, the gradient vectors are obtained with two pairs of operators: the first pair are $3 \times 3$ sized, that locates the gradient vectors at the center of each pixel, and the second pair are $4 \times 4$ sized, that locates them at the intersection of every 4 pixels. So, a more dense map of gradient vectors is computed.

Elliptical neighborhoods of different sizes have been considered for this analysis. These class of neighborhoods used in the edge detection algorithm in [7] have 7,13 and 19 gradient vectors inside them that are oriented depending on the direction of the central gradient vector of the straight edges used for this analysis. Figure 4 shows these elliptical neighborhoods for horizontal contours.

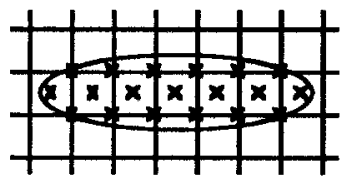

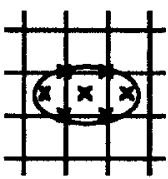

Figure 4

Different values of the SNR has been considered for this study. The standard deviation of the noise is fixed to 20 , and the contrasts of the contours analyzed are 1 , $2,4,8,12$ and 16 gray levels per pixel. So, the SNR are defined as $1 / 20,1 / 10,1 / 5$, $2 / 5,3 / 5$ and $4 / 5$ respectively. 
In order to compare the different analysis made in this work, the following quality factor is defined:

$$
\eta=\frac{n}{n^{\prime}+\left(n^{\prime}-n\right)}
$$

where $n: \quad$ Number of contour pixels detected.

$n$ : Number of contour pixels in the image.

$n "$ : Number of pixels detected as a contour (false detection included).

The values of $n, n^{\prime}$ and $n^{\prime \prime}$ are obtained as follows

$$
\begin{aligned}
& n=M \cdot p_{c}(i, N, t) \\
& n^{\prime}=M \cdot C \\
& n^{\prime \prime}=M \cdot\left[C \cdot p_{c}(i, N, t)+(1-C) p_{n c}(i, N, t)\right]
\end{aligned}
$$

where $M$ is the number of pixels in the image, and $C$ is the ratio of contour pixels in the image. The value of $p_{c}(i, N, t)$ depends on the analysis. In the case of the argument analysis $p_{c}(i, N, \alpha)$ is the probability that at least $i$ arguments of the $N$ gradients vectors in the ellipse fulfill $\left|\alpha_{j}-A\right| \leq \alpha$ when the central point of the ellipse is a contour pixel. $\alpha_{j}$ represents the set of the gradient vector arguments inside the ellipse. In the case of the module analysis $p_{c}(i, N, m)$ is the probability that at least $i$ modules of the $N$ gradients vectors in the ellipse fulfill $m_{j} \geq m$ when the central point of the ellipse is a contour pixel. $m_{j}$ represents the set of the gradient vector modules inside the ellipse. The value of $p_{n c}(i, N, \alpha)$ is similar to $p_{c}(i, N, \alpha)$ but it corresponds to the case when the central point of the ellipse is a non-contour pixel. These values are different for different values of SNR.

An example of these functions are shown in figure 5. Figure 5-a represents the probability function $p_{c}(13,13, \alpha)$ for the argument analysis, and figure 5-b a represents the probability function $p_{c}(13,13, m)$ for the module analysis. In both cases the SNR is $3 / 5$ and they are obtained in a heuristic analysis. The $X$ axis represents the threshold $\alpha$ and $m$ respectively.

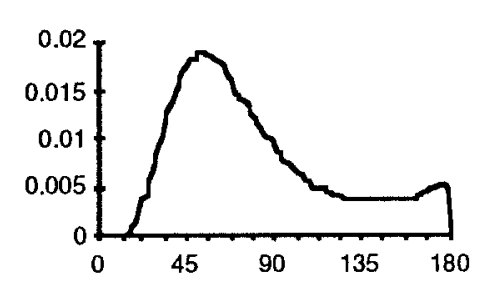

a

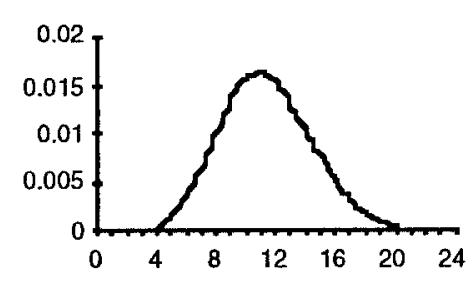

$\mathrm{b}$

Figure 5

Figure 6 represents an example of the quality factor. Figure 6-a for the argument condition and figure 6-b for the module condition. It corresponds to the same case as figure 5. The $\mathrm{Y}$ axis represents the quality factor and the $\mathrm{X}$ axis represents the threshold. A $10 \%$ of contour pixels in the image is assumed. So, $C=0.1$. 


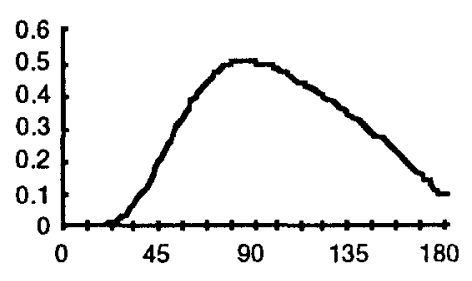

a

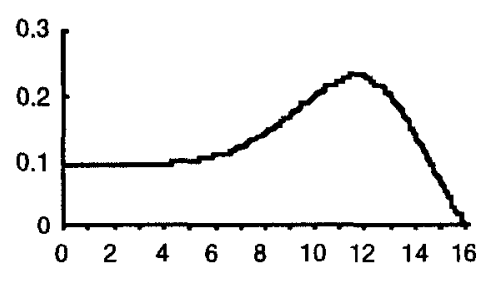

b

Figure 6

The most important value of these functions is the maximum and their thresholds. So, in the case of the argument the best threshold is $86^{\circ}$ where the quality factor is 0.506 , and the best threshold for the module is 12 where its quality factor is 0.232 . These are not the best quality factors for the elliptical neighborhood of size 13. For the argument the best quality factor is when at least 12 of the 13 gradient vectors $\left(p_{c}(12,13, \alpha)\right)$ fulfill the condition. In this case the quality factor is 0.536 with a threshold of $72^{\circ}$. With the module, the best quality factor is when at least 5 of the 13 gradient vectors $\left(p_{c}(5,13, m)\right)$ fulfill the condition. Here, the quality factor is 0.399 with a threshold of 6 .

Tables 1 shows the bests quality factors for different SNR, and different sizes of the elliptical neighborhood for the argument analysis, the module analysis, and the module and argument analysis. In each cell of the table there is the best quality factor that belongs to the best threshold, and in the case of the argument analysis and the module analysis how many gradient vectors must fulfill at least, the condition.

\begin{tabular}{|c|c|c|c|c|c|}
\hline & $\begin{array}{l}\text { Ellipse } \\
\text { Size }\end{array}$ & $\begin{array}{l}\text { Contrast }=4 \\
\text { SNR }=1 / 5\end{array}$ & $\begin{array}{l}\text { Contrast }=8 \\
\text { SNR }=2 / 5\end{array}$ & $\begin{array}{l}\text { Contrast }=12 \\
\text { SNR }=3 / 5\end{array}$ & $\begin{array}{c}\text { Contrast }=16 \\
\text { SNR }=4 / 5\end{array}$ \\
\hline \multirow{3}{*}{$\begin{array}{c}\text { Argument } \\
\text { Analysis }\end{array}$} & 7 & $\begin{array}{c}0.161 \\
(6 \text { from } 7)\end{array}$ & $\begin{array}{c}0.281 \\
(6 \text { from } 7)\end{array}$ & $\begin{array}{c}0.421 \\
(7 \text { from } 7)\end{array}$ & $\begin{array}{c}0.561 \\
(7 \text { from } 7)\end{array}$ \\
\hline & 13 & $\begin{array}{c}0.179 \\
(9 \text { from } 13)\end{array}$ & $\begin{array}{c}0.343 \\
(11 \text { from 13) }\end{array}$ & $\begin{array}{c}0.536 \\
\text { (12from 13) }\end{array}$ & $\begin{array}{c}0.705 \\
(12 \text { from 13) }\end{array}$ \\
\hline & 19 & $\begin{array}{c}0.202 \\
(14 \text { from 19) }\end{array}$ & $\begin{array}{c}0.409 \\
(15 \text { from } 19)\end{array}$ & $\begin{array}{c}0.636 \\
(15 \text { from } 19)\end{array}$ & $\begin{array}{c}0.805 \\
\text { (16 from 19) }\end{array}$ \\
\hline \multirow{3}{*}{$\begin{array}{l}\text { Module } \\
\text { Analysis }\end{array}$} & 7 & $\begin{array}{c}0.104 \\
(5 \text { from } 7)\end{array}$ & $\begin{array}{c}0.170 \\
(2 \text { from } 7)\end{array}$ & $\begin{array}{c}0.311 \\
(2 \text { from } 7)\end{array}$ & $\begin{array}{c}0.483 \\
(3 \text { from } 7)\end{array}$ \\
\hline & 13 & $\begin{array}{c}0.109 \\
\text { (5 from 13) }\end{array}$ & $\begin{array}{c}0.204 \\
(5 \text { from } 13)\end{array}$ & $\begin{array}{c}0.399 \\
(5 \text { from } 13)\end{array}$ & $\begin{array}{c}0.625 \\
\text { (6 from 13) }\end{array}$ \\
\hline & 19 & $\begin{array}{c}0.113 \\
(11 \text { from } 19)\end{array}$ & $\begin{array}{c}0.235 \\
(9 \text { from 19) }\end{array}$ & $\begin{array}{c}0.478 \\
(8 \text { from } 19)\end{array}$ & $\begin{array}{c}0.732 \\
(9 \text { from 19) }\end{array}$ \\
\hline \multirow{3}{*}{$\begin{array}{l}\text { Argument } \\
\text { and } \\
\text { Module } \\
\text { Analysis }\end{array}$} & 7 & 0.162 & 0.298 & 0.475 & 0.652 \\
\hline & 13 & 0.180 & 0.356 & 0.580 & 0.776 \\
\hline & 19 & 0.203 & 0.427 & 0.682 & 0.866 \\
\hline
\end{tabular}

Table 1

The conclusions of the results of this table are discussed in the next section. 


\section{Conclusions}

The most important conclusions of this work are the following:

- The argument of the gradient vector is more robust than the module for the detection of step edges. This means that the information of the argument allows to detect contours with lower contrast than the information of the module.

- The results of analyzing module and arguments together are only a little better, but the algorithm would be more complex than the other cases.

- The detection improves with the number of gradient vectors analyzed. The number of gradient vectors is limited because of the region to be analyzed must be over the contour. In curved lines the region to be analyzed must be curved too, in order to follow the same direction of the contour.

- Larger operators for the gradient vector obtention improve the detection of the contour, but it must be small to assure good location.

- Contours with SNR of $2 / 5$ are very difficult to detect. Contours with a SNR of $4 / 5$ can be detected with good results with the argument or the module information and an elliptical neighborhood of size 13.

\section{References}

[1] Kim L. Boyer, Sudeep Sarkar, "On the Localization Performance Measure and Optimal Edge Detection," IEEE Trans. on PAMI Vol. 16 No. 1 (106-110) January 1994.

[2] J. Brian Burns, Allen R. Hanson, Edward M. Riseman, "Extracting Straight Lines," Computer and Information Science Dept. Tech. Rep. 84-29, Univ. of Massachusetts, December 1984.

[3] John F. Canny, "Finding Edges and Lines in Images," MIT., Cambridge, Tech. Rep. 720, June 1983.

[4] L. S. Davis, "A Survey of Edge Detection Techniques," Comp. Graph. and Image Proc., 4 (248-270) 1975.

[5] R. Deriche, "Using Canny's Criteria to Derive a Recursive Implemented Optimal Edge Detector," The Int. Jour. of Comp. Vision, 1 (167-187), 1987.

[6] Robert M. Haralick, "Digital Step Edges from Zero Crossing of Second Directional Derivatives," IEEE Trans. on PAMI Vol. 6 No.1 (58-68) Jan.1984.

[7] Albert Larré, Eduard Montseny, "A Step Edge Detector Algorithm Based On Symbolic Analysis, " 12th IAPR Inter. Conf. on Pattern Recognition, Vol. 1 (610) October 1994.

[8] D. C. Marr, E. Hildreth, "Theory of Edge Detection," Proc. of the Royal Society of London, Series B, Vol. 2071980.

[9] V. S. Nalwa, T. O. Binford, "On Detecting Edges," IEEE Trans. on PAMI Vol. 8 No.6 (699-714) November 1986.

[10] Azriel Rosenfeld, M. Thurston, Y. Lee, "Edge and Curve Detection: Further Experiments," IEEE Trans. on Comp., Vol.C-21 No.7 (677-710) July 1972.

[11] Vincent Torre, Tomaso Poggio "On edge detection," IEEE Trans.PAMI Vol. 8 No.2 (147-163) February.1986. 